\title{
Zonação de comunidade bêntica do entremarés em molhes sob diferente hidrodinamismo na costa norte do estado do Rio de Janeiro, Brasil
}

\author{
Bruno P. Masi \& Ilana R. Zalmon \\ Laboratório de Ciências Ambientais, Universidade Estadual do Norte Fluminense. Avenida Alberto Lamego 2000, 28013-602 \\ Campos, Rio de Janeiro, Brasil. E-mail: bmasibio@yahoo.com.br; ilana@uenf.br
}

\begin{abstract}
Zonation of intertidal benthic communities on breakwaters of different hydrodynamics in the north coast of the state of Rio de Janeiro, Brazil. The present study aims to compare the vertical distribution of intertidal benthic communities in two sites composed by granitic boulders with distint hydrodynamics due to different wave swells at Farol de São Tomé (Pier) and Barra do Furado beaches (Barra), both in northern state of Rio de Janeiro. Quadrats of $400 \mathrm{~cm}^{2}$ were overlapped along three vertical profiles on each site and were sampled by the photoquadrat method from $0.2 \mathrm{~m}$ of the tide level to the upper limit of Littorina spp. The upper limit of the marine organisms was higher at Barra site $(3.8 \mathrm{~m})$ than at Píer site $(2.2 \mathrm{~m})$. Nine species were common to both sites. Chaetomorpha sp., Chondracanthus teedii (Mertens ex Roth) Fredericq, and Grateloupia sp. were unique to Barra, whereas Tetraclita stalactifera (Lamarck, 1818), Fissurella clench, Gracilaria domingensis (Kützing) Sonder ex Dickie, and Hypnea musciformis (Wulfen in Jacqu.) Lamouroux occurred only at Pier boulders. On both sites species richness and diversity were superior at the intermediate quadrats of the intertidal zone. The highest values were recorded at Barra. The most pronounced assemblage differences between equivalent areas of either site occurred on the lower upper eulittoral band followed by the lower eulittoral and the sub-littoral fringe, respectively. The littoral fringe assemblage was the only one that did not show significant differences among the studied sites, yet a larger range of this fringe and the upper eulittoral band at Barra was quite evident. This site was mostly characterized by species of more exposed areas, such as Chaetomorpha sp. and Perna perna (Linnaeus, 1758) on upper and lower eulittoral bands, and by $C$. teedii and Ulva fasciata Delile, 1813 on the sub-littoral fringe. At Píer, the intertidal zone showed an even distribution, reflecting a less stressful environment. The differences on vertical distribution of the local benthic, particularly in the extent of the upper bands, indicate the variable conditions of wave exposure.
\end{abstract}

KEY WORDS. Benthic assemblage; boulders; spatial variability; vertical distribution.

RESUMO. O presente estudo pretende comparar a distribuição vertical da comunidade bêntica na zona entremarés em dois locais compostos por matacões graníticos caracterizados por hidrodinâmica distinta, reflexo da diferença na orientação dos molhes nas praias do Farol de São Tomé (Píer) e na Barra do Furado (Barra), norte do Estado do Rio de Janeiro. Quadrados de $400 \mathrm{~cm}^{2}$ foram sobrepostos ao longo de três perfis verticais de ambos os sítios e amostrados através do método de fotoquadrats, desde o nível $0,2 \mathrm{~m}$ da maré até o limite superior de Littorina spp. O limite superior dos organismos marinhos foi ampliado na Barra $(3,8 \mathrm{~m})$ em relação ao Píer $(2,2 \mathrm{~m})$. Quanto à composição taxonômica, nove espécies foram comuns. Chaetomorpha sp., Chondracanthus teedii (Mertens ex Roth) Fredericq e Grateloupia sp. foram exclusivas na Barra, enquanto Tetraclita stalactifera (Lamarck, 1818), Fissurella clench, Gracilaria domingensis (Kützing) Sonder ex Dickie e Hypnea musciformis (Wulfen in Jacqu.) Lamouroux ocorreram somente no Píer. Em ambos os locais, a riqueza e a diversidade de espécies foram superiores nos quadrados intermediários. Os maiores valores foram registrados na Barra. As maiores diferenças nos agrupamentos entre faixas equivalentes de locais distintos ocorreram na faixa eulitorânea superior, seguida pela faixa eulitorânea inferior e franja sublitorânea. Apenas a orla litorânea não revelou diferença significativa entre os locais, mas uma maior extensão desta franja e da faixa eulitorânea superior era bastante evidente. As demais faixas na Barra do Furado foram caracterizadas em grande parte por espécies típicas de ambientes mais expostos como Chaetomorpha sp. na faixa eulitorânea superior e Perna perna (Linnaeus, 1758) na eulitorânea inferior, além de C. teedii e Ulva fasciata Delile, 1813 na franja sublitorânea. No Píer, as diferentes faixas apresentavam distribuição equïitativa, refletindo um ambiente menos estressante. As diferenças observadas na distribuição vertical dos organismos bênticos, principalmente na extensão das faixas superiores, evidenciam condições de exposição a ondas variáveis.

PALAVRAS-CHAVE. Agrupamentos bênticos; distribuição vertical; matacões; variabilidade espacial.

Revista Brasileira de Zoologia 25 (4): 662-673, December, 2008 
A distribuição vertical da comunidade bêntica de costões rochosos na região entremarés é caracterizada pela distribuição de organismos ou associações de organismos em zonas dispostas horizontalmente (STEPHENSON \& STEPHENSON 1949, Lewis 1964). A variabilidade no eixo vertical do costão geralmente ocorre em uma escala de centímetros ou poucos metros. Diversos fatores abióticos (e.g. variação do nível de maré, grau de exposição às ondas, inclinação do substrato, dessecação e degelo) e bióticos (e.g. predação, herbivoria, competição) são conhecidos por afetar a distribuição vertical dos organismos na zona entremarés (revisado por BoAden \& Seed 1985, Moore \& Seed 1985, Little \& Kitching 1996, Benedetti-Cecchi 2000).

Ao longo do eixo horizontal do costão, a variabilidade espacial tem sido um tema vastamente citado na literatura (Underwood 1981, Underwood \& Chapman 1998a, b, BenedetTICecchi \& Cinelli 1997, Guichard et al. 2001, Araújo et al. 2005), estando relacionada a uma escala específica de observação. UNDERWOOD \& CHAPMAN (1998a, b) observaram uma variabilidade espacial considerável em todas as associações de organismos examinadas em diferentes escalas (i.e. metros, dezenas de metros e quilômetros) em costões rochosos abrigados na Austrália. Araújo et al. (2005) citam que na costa portuguesa a variabilidade horizontal em grande escala (quilômetros) está relacionada ao grau de exposição às ondas, enquanto que em escala de poucos metros tal variabilidade está relacionada à heterogeneidade do habitat (veja também BenedetTi-Cecchi \& Cinelli 1997). Segundo Underwood (1981), em uma escala de poucos metros a variabilidade também pode estar relacionada ao grau de exposição às ondas.

Diferenças na comunidade bêntica em costões submetidos a diferentes condições de exposição às ondas são conhecidas desde Lewis (1964), que considerou o efeito desta ação na altura e largura das faixas horizontais dos organismos. Uma costa mais aberta está sujeita a um batimento mais forte de ondas em relação a uma área mais abrigada. A influência das ondas na estrutura da comunidade entremarés tem sido abordada considerando mudanças na composição da comunidade (LEwIs 1964), ampliação da zona entremarés (LEWIS 1964, UNDERWOOD 1981), riqueza e diversidade de espécies (UNDERWOOD 1981, THOMAs 1985, Boaventura et al. 2002, Araújo et al. 2005), dinâmica das "manchas" de organismos (MENGE et al. 2005) e na influência relativa sobre a predação e competição (Good 2004).

A complexidade topográfica de um substrato é uma característica física particularmente importante na região entremarés onde os rigores da ação mecânica das ondas e a dessecação são importantes (JACOBI \& LANGEvin 1996). A heterogeneidade do substrato pode modificar o padrão hidrodinâmico específico durante a maré alta e influenciar no sombreamento e na intensidade de vento durante a maré baixa (Guichard et al. 2001).

A adição de estruturas artificiais na costa tem ocorrido devido ao desenvolvimento costeiro no mundo. Molhes ou quebra-mares (breakwaters) têm sido utilizados na construção de marinas e portos e na proteção de praias arenosas contra a erosão. Tais hábitats são tridimensionais, oferecendo uma variedade de ambientes diferentes quanto à orientação, sombreamento e grau de exposição às ondas na região entremarés (BULLERI \& Chapman 2004). A variabilidade horizontal em pequena escala nestes locais é ainda mais evidente face à maior heterogeneidade espacial e conseqüente incremento na complexidade.

A variabilidade horizontal e vertical em molhes distantes em algumas centenas de metros pode ser incrementada se os mesmos apresentarem diferentes orientações em relação às ondulações (wave swell), o que acarreta padrões de hidrodinamismo diferenciados (Bulleri \& CHAPMAN 2004). Os autores supracitados atribuíram as diferenças entre associações de organismos em diversas superfícies às variações na estrutura do hábitat, excluindo o fator exposição às ondas por possuírem a mesma orientação quanto à ondulação.

O presente estudo pretende caracterizar comparativamente a comunidade bêntica do entremarés ao longo do gradiente vertical em dois molhes na costa norte do Estado do Rio de Janeiro, com diferentes intensidades de exposição às ondas, reflexo da diferença na orientação dos molhes, o que causa alteração no padrão hidrodinâmico. Dessa forma, este estudo testa a hipótese de que faixas verticais caracterizadas por associações particulares de organismos bênticos da zona entremarés diferem entre sítios em função do grau de exposição às ondas, variável quanto à orientação dos molhes de cada local.

\section{MATERIAL E MÉTODOS}

O estudo foi desenvolvido na costa norte do estado do Rio de Janeiro, em dois molhes localizados nas praias da Barra do Furado e do Farol de São Tomé $\left(22^{\circ} 02^{\prime} S, 41^{\circ} 03^{\prime} \mathrm{W}\right)$, distantes entre si cerca de dois quilômetros, aqui referidos como Barra e Píer, respectivamente. Estes são constituídos por matacões graníticos transplantados, apresentando uma superfície irregular e descontínua formando "degraus" (Figs 1 e 2). Ambos os molhes apresentam uma inclinação total de $50^{\circ}$, mas não apresentam a mesma orientação quanto à ondulação (wave swell) (Fig. 3). As principais conseqüências são as diferenças no grau de exposição e alterações no padrão hidrodinâmico. $\mathrm{O}$ vento nordeste é predominante na região durante o ano.

Na Barra, os matacões ocorrem a partir da praia e possuem uma área total aproximada de $80 \mathrm{~m}$ de comprimento. A amostragem foi realizada na superfície rochosa sudoeste, perpendicular à linha da costa (Fig. 1).

No Píer, os matacões distam $10 \mathrm{~m}$ da praia e possuem uma área total aproximada de $30 \mathrm{~m}$ de comprimento. O programa de amostragem foi realizado na superfície rochosa oeste, onde a ação das ondas é menos intensa devido à proteção da superfície rochosa em relação à ondulação (Fig. 2).

Para mensurar e expressar o grau de exposição às ondas na superfície rochosa de cada molhe foi avaliado o tamanho das ondas com uma régua de $2,0 \mathrm{~m}$ (escala $0,5 \mathrm{~m}$ ), distante $50 \mathrm{~m}$ do observador, e o período das ondas que corresponde ao intervalo 


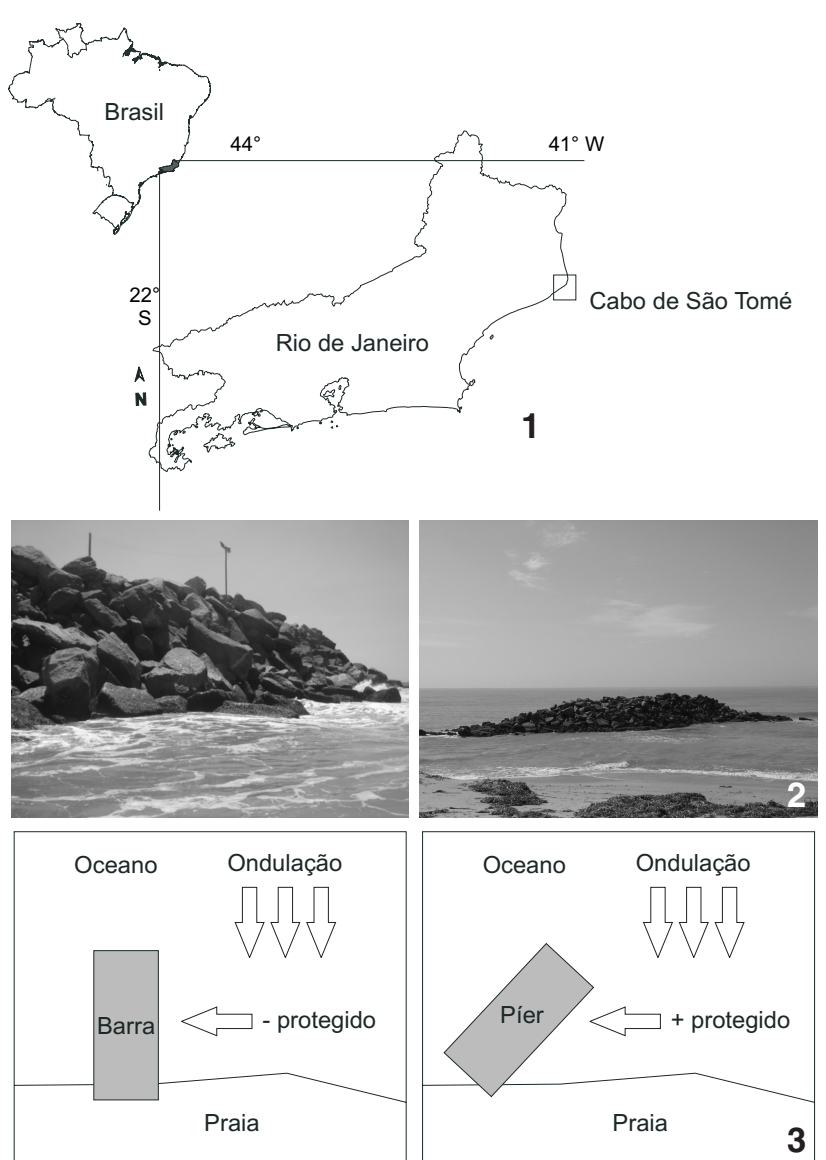

Figuras 1-3. Localização dos locais de estudo na costa norte do estado do Rio de Janeiro: (1) Barra do Furado (Barra), (2) Farol de São Tomé (Píer), (3) Esquema representando a orientação dos molhes Píer e Barra em relação à costa, refletindo em diferentes condições de exposição às ondas visto à direção predominante da ondulação.

de tempo em segundos decorrido entre a passagem sucessiva de duas cristas por um ponto fixo. Os resultados dos parâmetros investigados foram comparados à tabela proposta por HARPER et al. (1991) obtendo-se uma classificação (muito protegido, protegido, semi-protegido, semi-exposto e exposto) e uma escala de 1 a 10, respectivamente de muito protegido à exposto.

O programa de amostragem foi realizado em ambos os locais em julho de 2005, na parte da manhã em baixamar. Os organismos foram previamente coletados para a identificação taxonômica da comunidade. Os cirripédios Chthamalus proteus Dando \& Southward, 1980 e C. bisinuatus Pilsbry, 1916 foram caracterizados ao nível de gênero devido à impossibilidade de distingüi-los.

Três perfis verticais de $4 \mathrm{~m}$ de largura, distantes cerca de $6 \mathrm{~m}$, foram amostrados em cada local, cada um representando uma unidade amostral. Uma câmera digital Cannon PowerShot A510 4.1 Mp em compartimento estanque foi utilizada fixada a uma estrutura de PVC com 20 X $20 \mathrm{~cm}$ de lado para a metodologia de fotoquadrat (PreskitT et al. 2004). MACEDo et al. (2006) compararam a metodologia convencional em campo e por fotoquadrat neste mesmo local e verificaram que para estudos de zonação bêntica a amostragem por fotografia digital é bastante eficiente, acurada e vantajosa em relação ao método de amostragem in situ. Em cada perfil, os quadrats foram fotografados desde o nível 0,2 $\mathrm{m}$ da maré, até um ponto selecionado acima do último organismo marinho na porção superior do substrato rochoso (geralmente Littorina spp.). Na Barra foi necessário um maior número de quadrados para amostragem da zona entremarés ( $\mathrm{n}=18$ quadrados na Barra e $\mathrm{n}=10$ quadrados no Píer), face à sua maior extensão.

As amostragens foram realizadas em superfícies relativamente perpendiculares e voltadas para o mar. Cada fotografia foi analisada através do software CPCe V3.4 (Coral Point Count with Excel extensions), a partir de um grid com 100 pontos de interseção (KOHLER \& GILl 2006). A distinção entre a cobertura do estrato primário e secundário não foi considerada. Devido à descontinuidade do substrato, a determinação de cada altura foi executada por seções, utilizando-se o método de GeverTz (1995).

Os níveis de maré foram obtidos através da tábua de marés para o Terminal Marítimo de Imbetiba, disponível em: www. mar.mil.br/dhn/chm.

Os agrupamentos bênticos nas diferentes alturas foram estimados através da composição taxonômica, riqueza de espécies (número total e médio), diversidade de Brillouin (ZAR 1984) e porcentagem de cobertura média nos três perfis em cada local estudado.

A análise comparativa dos dois locais estudados em relação às associações de organismos bênticos nas diferentes alturas incluiu análise de agrupamento e o método de ordenamento MDS, utilizando-se o coeficiente de similaridade de Bray-Curtis com os dados de porcentagem de cobertura média das espécies. A confiabilidade do dendrograma foi avaliada pelo coeficiente de correlação cofenético, que segundo VALENTin (2000) é aceitável acima do valor 0,8 . A adequação da configuração das amostras no ordenamento MDS foi obtida através do valor de estresse, que abaixo de 0,05 proporciona uma excelente representação espacial sem probabilidade de má interpretação (CLARKE \& WARWICK 2001).

O teste de permutação ANOSIM (one way) foi empregado a fim de avaliar a significância das diferenças entre os grupos prédefinidos a partir da análise de agrupamento representadas no método de ordenamento MDS. As matrizes de similaridade incluíram a porcentagem de cobertura dos organismos presentes em cada local. As três unidades amostrais de mesma altura e local foram tratadas como réplicas para aumentar a possibilidade de permutações e, conseqüentemente, o poder do teste (Clarke \& WARWICK 2001). O teste ANOSIM produz uma estatística R que varia em uma amplitude de $-1 \mathrm{a}+1$. Valores $\mathrm{R}$ iguais $\mathrm{a}+1$ são obtidos apenas quando todas as réplicas dentro dos grupos são mais similares entre si do que qualquer réplica de grupos diferentes. 
O procedimento de porcentagem de similaridades (SIMPER) definiu o percentual de contribuição das espécies dentro e entre os grupos evidenciados pela análise de agrupamento e representadas no método de ordenação MDS (CLARKE \& WARWICK 2001). Uma contribuição cumulativa de $80 \%$ foi aplicada como em BoAventura et al. (2002).

A análise de dados foi realizada com o programa PRIMER 6 (ClaRke \& WARWick 2001) e o coeficiente cofenético calculado como software NSTSYS pc 2.1. Representações gráficas do padrão de distribuição vertical das principais espécies na região entremarés nos molhes estudados de ambos os locais são indicadas em diagramas com os níveis de marés e utilizando-se a terminologia de Lewis (1964) adaptado por PaUla (1987)

\section{RESULTADOS}

O grau de exposição às ondas nas superfícies dos molhes estudados foi diferenciado. O critério período médio das ondas na Barra $(5,8 \pm 1,6)$ classificou o local como semi-exposto (escala 8) enquanto que o Píer $(1,0 \pm 0,6)$ foi classificado como muito protegido (escala 1 ). O critério altura das ondas classificou a Barra $(2,0 \pm 1,2)$ como semi-exposto (escala 8), enquanto que o Píer $(1,0 \pm 0,6)$ foi classificado como semi-protegido (escala 6). Considerando os parâmetros das ondas mensurados, a Barra apresentou uma maior grau de exposição às ondas quando comparado com o Píer.

A riqueza total foi semelhante em ambos os molhes, com 12 espécies registradas no Píer e 13 na Barra (Tab. I). A presença ou ausência de espécies particulares mostra a afinidade de cada qual a um grau diferenciado de ação das ondas. Como espécies exclusivas destacaram-se Chaetomorpha sp., Chondracanthus teedii e Grateloupia sp. - Barra - e Tetraclita stalactifera (Lamarck, 1818), Fissurella clench, Gracilaria domingensis (Kützing) Sonder ex Dickie e Hypnea musciformis (Wulfen in Jacqu.) Lamouroux - Píer.

A representação gráfica da distribuição vertical das espécies mais representativas revela diferenças na sua amplitude vertical e abundância relativa em ambos os locais estudados (Figs 413). Ao longo do eixo vertical dos molhes, as espécies colonizam níveis mais elevados do substrato na Barra em relação ao Píer.

A mesma tendência de declínio unidirecional de espaço vazio para a porção inferior da zona entremarés foi registrada no Píer e na Barra (Fig. 4). O gastrópode Littorina ziczac (Gmelin, 1791), apesar do baixo valor médio de cobertura (Barra: 3,0\%; Píer: 5,7\%), caracterizou o limite superior da zona entremarés (Fig. 5). O cirripédio Chthamalus sp. apresentou os maiores valores médios de cobertura na porção superior da zona entremarés (Barra: 21,3\%; Píer: 63,7\%) (Fig. 6). O gastrópode herbívoro, Collisella subrugosa (Orbigny, 1846), apresentou uma ampla faixa de distribuição vertical na Barra, estendendo-se da porção superior a média da zona entremarés, bastante similar em extensão a clorófita Chaetomorpha sp., ausente no Píer (Figs 7 e 8). O bivalve Perna perna (Linnaeus, 1758) apresentou uma distribuição vertical descontínua no Píer, restringindo-se à porção inferior, enquanto na Barra foi mais abundante, ocupando in-
Tabela I. Lista de espécies identificadas na região entremarés nos molhes da Barra e Píer, costa norte do Estado do Rio de Janeiro.

\begin{tabular}{lcc}
\hline \multicolumn{1}{c}{ Espécies } & Barra & Píer \\
\hline Chthamalus spp. & + & + \\
Tetraclita stalactifera (Lamarck, 1818) & - & + \\
Collisella subrugosa (Orbigny, 1846) & + & + \\
Fissurella clenchi (Farfante, 1943) & - & + \\
Littorina ziczac (Gmelin, 1791) & + & + \\
Phragmatopoma lapidosa Kinberg (1867) & + & + \\
Brachidontes solisianus (Linnaeus, 1758) & + & + \\
Perna perna (Linnaeus, 1758) & + & + \\
Chaetomorpha sp. & + & - \\
Ulva fasciata Delile, 1813 & + & + \\
Centroceras clavulatum (Agardh) Montagne & + & + \\
Chondracanthus teedii (Mertens ex Roth) Fredericq & + & - \\
Gracilaria domingensis (Kützing) Sonder ex Dickie & - & + \\
Grateloupia sp. & + & - \\
Gymnogongrus griffithsiae (Turner) Martius & + & + \\
Hypnea musciformis (Wulfen in Jacqu.) Lamouroux & - & + \\
\hline Total & 12 & 13 \\
\hline (+) Presença, (-) ausencia. & &
\end{tabular}

(+) Presença, (-) ausência.

clusive a porção média da zona entremarés (Fig. 9). A rodófita Centroceras clavuatum ocupou, como os demais organismos, uma posição superior na Barra, com cobertura média inferior em relação ao Píer (Barra: 5\%; Píer: 23,3\%) (Fig. 10). Dentre as espécies mais representativas da porção inferior da zona entremarés de ambos os locais, a clorófita Ulva fasciata Delile, 1813 apresentou uma faixa de distribuição mais ampla, atingindo a porção superior dos molhes na Barra (Fig. 11). Já a poliqueta Phragmatopoma lapidosa Kinberg (1867), representada pelos "recifes de areia" que constrói, foi mais abundante no extremo inferior da zona entremarés na Barra (58,7\%), enquanto no Píer os valores médios de cobertura apresentaram-se mais homogêneos em toda a faixa inferior, em torno de $45 \%$ (Fig. 12).

Em ambos os locais, o número de espécies foi superior nos quadrados intermediários (Barra: B8 à B12 = 1,6 a 2,6 m; Píer: P5 e P6 = 1,0 a 1,4 m), com cinco a sete espécies em média e, inferior nos quadrados extremos (Fig. 13). Nestes, a Barra apresentou uma variação mais ampla no número total de espécies (1 a 7), enquanto no Píer a variação foi de duas a quatro espécies.

A diversidade apresentou uma tendência similar ao número de espécies, com valores médios superiores nos quadrados intermediários em ambos os locais de estudo (Barra: B7 à B12 = 1,4 a 2,6 m; Píer: P5 e P6 = 1,0 a 1,4 m). Comparando-se os diferentes locais, na Barra foram registrados os maiores valores médios de diversidade (Barra: $\mathrm{H}=1,3$ em B9; Píer: $\mathrm{H}=1,1$ em P5) e diversidade máxima (Barra: 1,7 em B10; Píer: 1,4 em P5). Vale ressaltar as expressivas variações de riqueza e diversidade no sítio Barra em uma mesma altura (Fig. 14). 

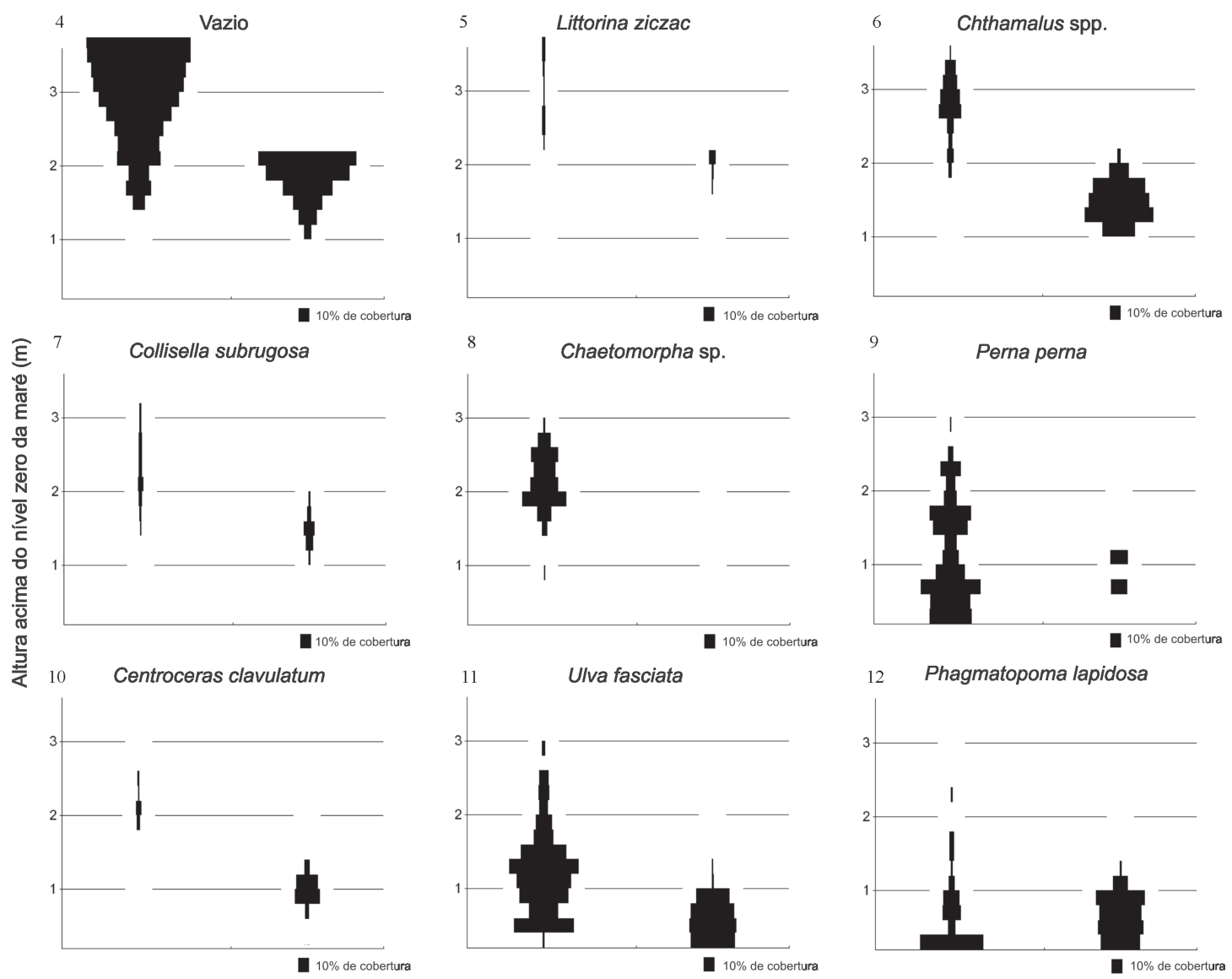

Figuras 4-12. Distribuição vertical das espécies mais representativas nos molhes Barra (coluna da esquerda) e Píer (coluna da direita), costa norte do Estado do Rio de Janeiro.

A análise de agrupamento das associações bênticas nas diferentes alturas revelou na Barra a formação de cinco grupos principais com $50 \%$ de similaridade, que corresponderam a cinco faixas da zona entremarés (Fig. 15): IB, superior, caracterizada por L. ziczac; IIB, intermediária superior, predominada por Chthamalus spp.; IIIB, intermediária média, representada por Chaetomorpha sp., P. perna e U. fasciata; IVB, faixa intermediária inferior, predominada por $U$. fasciata e P. perna; VB, inferior, com maior abundância de Phragmatopoma lapidosa e $P$. perna. O valor do coeficiente cofenético foi maior do que o aceitável $(\mathrm{r}=0,80)$, refletindo a confiabilidade do dendrograma.

No Píer também se verificou a formação de cinco grupos principais com 50\% de similaridade (Fig. 15): IP, faixa superior predominada por L. ziczac; IIP e IIIP, faixas intermediárias superior e média, caracterizada por Chthamalus spp.; IVP, faixa intermediária inferior, representada por Chthamalus spp., $P$. lapidosa, Centroceras clavulatum (Agardh) Montagne e Colisella subrugosa; VP, faixa inferior com maior abundância de $U$. fasciata e P. lapidosa. O valor do coeficiente cofenético também foi maior do que o aceitável $(\mathrm{r}=0,80)$.
A reunião dos quadrados (alturas) na análise de ordenação MDS correspondeu ao padrão gerado pela análise de agrupamento para ambos os locais (Fig. 16). A representação gráfica do método evidencia uma seqüência ordenada das alturas formando um continuum ao longo de ambos os substratos investigados. Os valores do estresse para a configuração bidimensional do MDS de 0,03 para a Barra e de 0,01 para o Píer foi baixo, indicando que as similaridades estão adequadamente representadas pelas distâncias no plano, denotando uma representação gráfica adequada.

A variabilidade espacial vertical em pequena escala $(0,2$ a $3 \mathrm{~m}$ ) das associações de organismos entre as diferentes alturas (quadrados) em cada praia, avaliada através da análise de similaridade (ANOSIM), revelou diferenças significativas ao longo do eixo vertical dos molhes na Barra $(R=0,57, p=0,1 \%)$ e no Píer $(R=0,66, p=0,1 \%)$. O teste de permutação ANOSIM também revelou diferença significativa entre os grupos (faixas da zona entremarés) formados a partir da análise de agrupamento tanto na Barra $(\mathrm{R}=0,75 ; \mathrm{p}=0,1 \%)$ como no Píer $(\mathrm{R}=$ 0,$87 ; \mathrm{p}=0,1 \%$ ). 


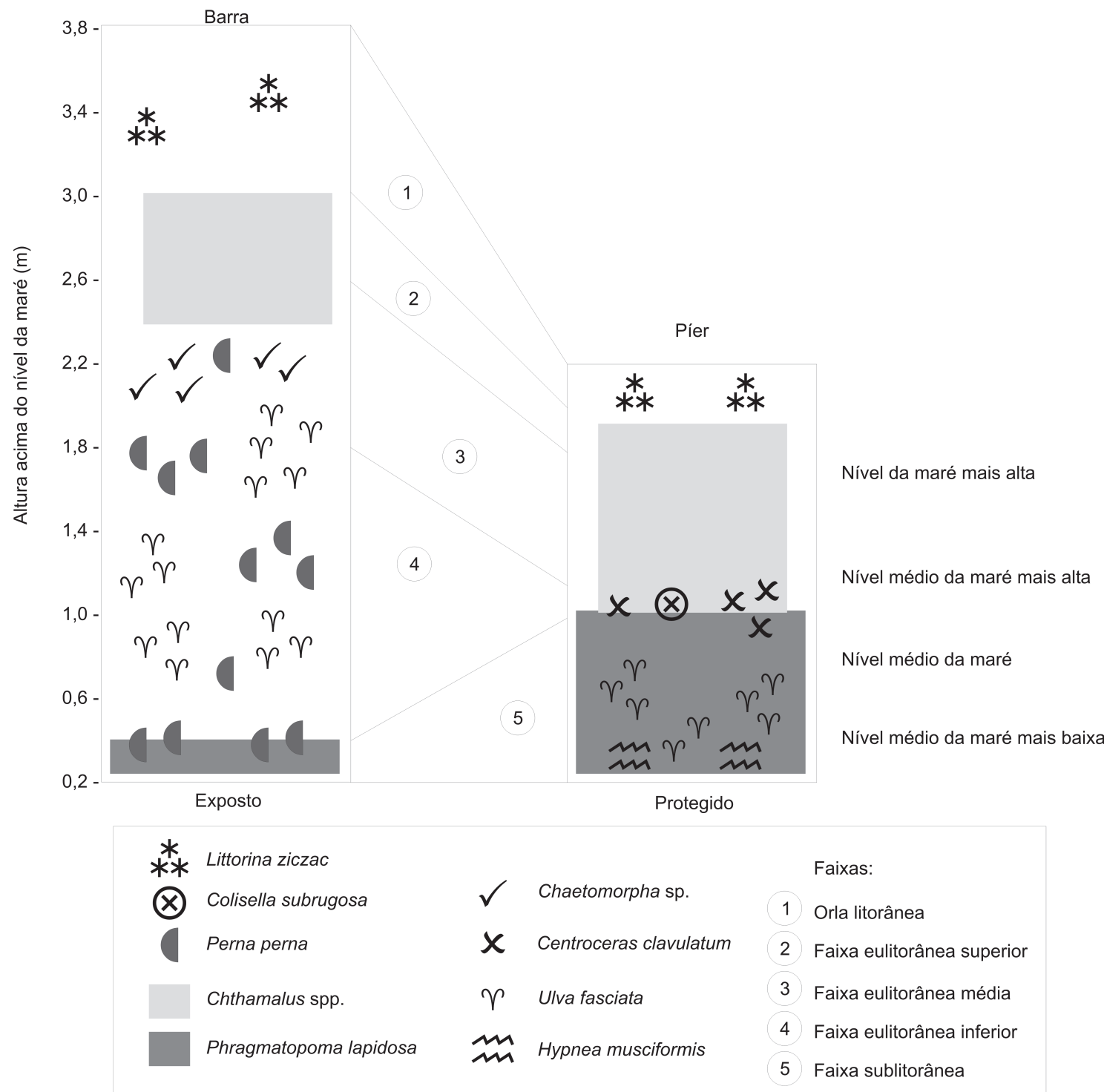

Figura 13. Distribuição vertical dos principais organismos dos molhes Barra (exposto) e Píer (protegido) incluindo os níveis de maré calculados para o período de 30 dias antes da amostragem.

Ao examinar a hipótese de diferenças significativas entre faixas equivalentes de locais distintos, a análise de similaridade ANOSIM evidenciou as maiores diferenças entre as faixas III, IV e V, respectivamente (Tab. II). Apenas as comparações dos grupos I e II da Barra com os grupos I e II do Píer (faixas mais superiores de ambos os molhes) não apresentaram valores significativos (IB vs IP: $\mathrm{R}=-0,193 ; \mathrm{p}=88,1 \%$; IIB $v s$ IIP: $\mathrm{R}=-0,267 ; \mathrm{p}=94,3 \%)$.

A análise SIMPER definiu as espécies que mais contribuíram para a similaridade dentro e entre os grupos formados na análise de agrupamento para os dois locais estudados (Tabs III
Tabela II. ANOSIM. Resultados do teste pareado entre grupos equivalentes para os molhes da Barra (B) e Píer (P).

\begin{tabular}{lcc}
\hline Grupos & Estatística $\mathrm{R}$ & $\mathrm{p}(\%)$ \\
\hline IB vs IP & $-0,193$ & $88,10 \mathrm{~ns}$ \\
IIB vs IIP & $-0,267$ & $94,30 \mathrm{~ns}$ \\
IIIB vs IIIP & 0,803 & 0,02 * \\
IVB vs IVP & 0,702 & $0,40 *$ \\
VB vs VP & 0,503 & $0,40 *$ \\
\hline
\end{tabular}

${ }^{\star} p<5 \%$, (ns) não significativo. 

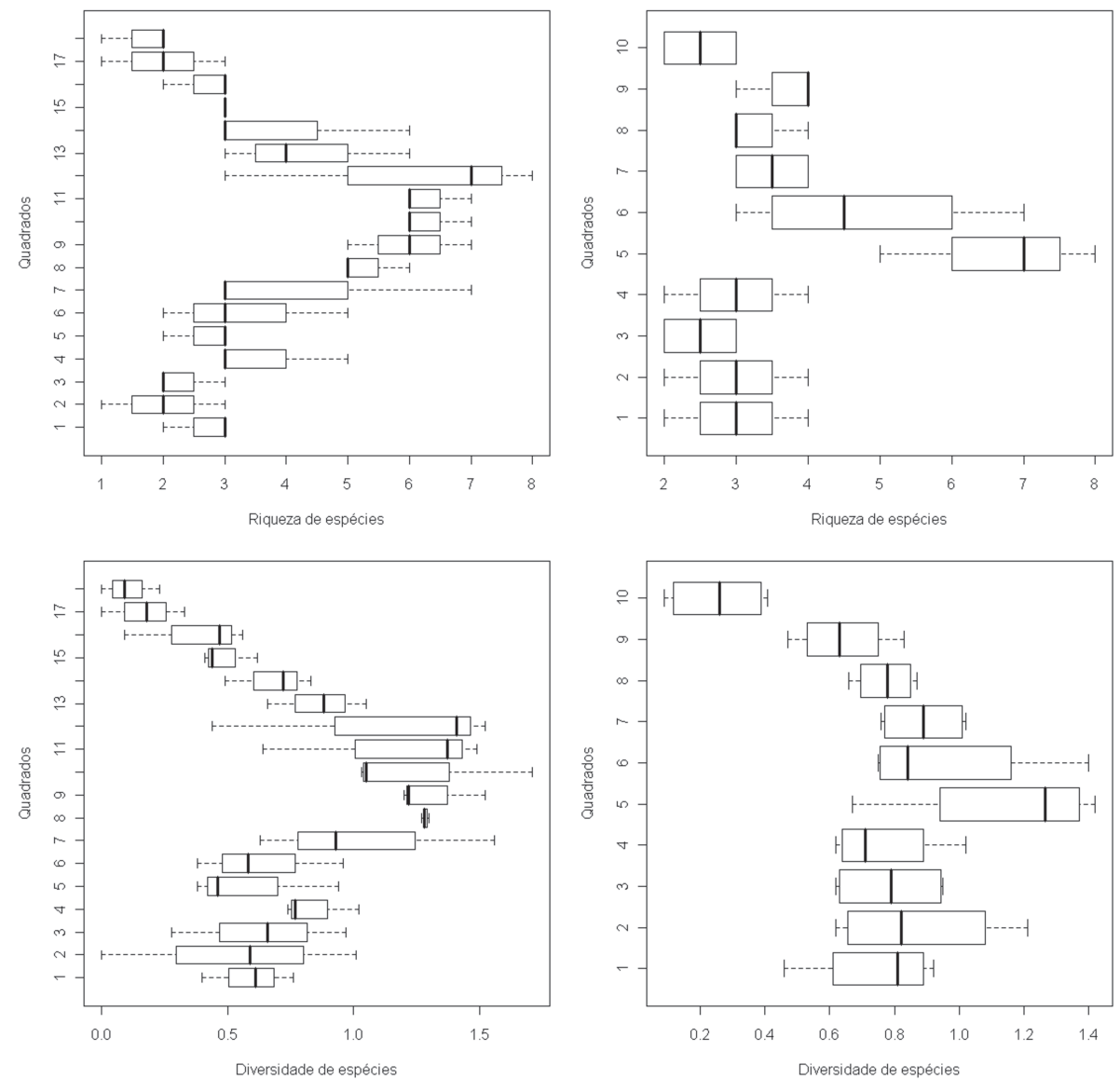

Figura 14. Riqueza e diversidade de espécies na região entremarés nos dois locais estudados (B1 e P1: quadrado inferior nos molhes Barra e Píer = 0,2 a 0,4 m; P10: quadrado superior no Píer = 2,0 a 2,2 m; B18: quadrado superior na Barra: 3,6 a 3,8 m). Valores médios de três perfis.

Tabela III. Análise SIMPER com a contribuição das espécies para a similaridade dentro dos grupos e para a dissimilaridade entre os grupos resultantes da análise de agrupamento na Barra.

\begin{tabular}{lccccc}
\hline $\begin{array}{c}\text { Similaridade dentro } \\
\text { dos grupos (\%) }\end{array}$ & IB & IIB & IIIB & IVB & VB \\
\hline Littorina ziczac & 100,00 & & & & \\
Chthamalus spp. & & 90,96 & & & \\
Chaetomorpha sp. & & & 50,68 & & \\
Perna perna & & & 14,44 & 37,94 & 32,61 \\
Ulva fasciata & & & 14,99 & 56,56 & \\
Phragmatopoma lapidosa & & & & & 65,76 \\
\hline Total & 100,00 & 90,96 & 80,10 & 94,59 & 98,37 \\
\hline
\end{tabular}

e IV). Em ambos, Littorina ziczac foi a espécie responsável pelo Grupo I (quadrados superiores) e Chthamalus pelo Grupo II (quadrados intermediários superiores). No Grupo III (quadrados intermediários médios), Chaetomorpha sp., $U$. fasciata e $P$. perna
Tabela IV. Análise SIMPER com a contribuição das espécies para a similaridade dentro dos grupos e para a dissimilaridade entre os grupos resultantes da análise de agrupamento no Píer.

\begin{tabular}{lccccc}
\hline $\begin{array}{c}\text { Similaridade dentro } \\
\text { dos grupos (\%) }\end{array}$ & IP & IIP & IIIP & IVP & VP \\
\hline Littorina ziczac & 84,43 & & & & \\
Chthamalus spp. & & 89,79 & 89,59 & 31,29 & \\
Phragmatopoma lapidosa & & & & 24,48 & 50,84 \\
Centroceras clavulatum & & & & 22,48 & \\
Colisella subrugosa & & & & 10,97 & \\
Ulva fasciata & & & & & 43,76 \\
\hline Total & 84,43 & 89,79 & 89,59 & 89,23 & 94,60 \\
\hline
\end{tabular}

foram as espécies mais comuns na Barra, enquanto apenas Chthamalus spp., predominou no Píer. No Grupo IV (quadrados intermediários inferiores), $P$. perna e $U$. fasciata foram a espécies mais comuns na Barra, enquanto no Píer, Chthamalus 

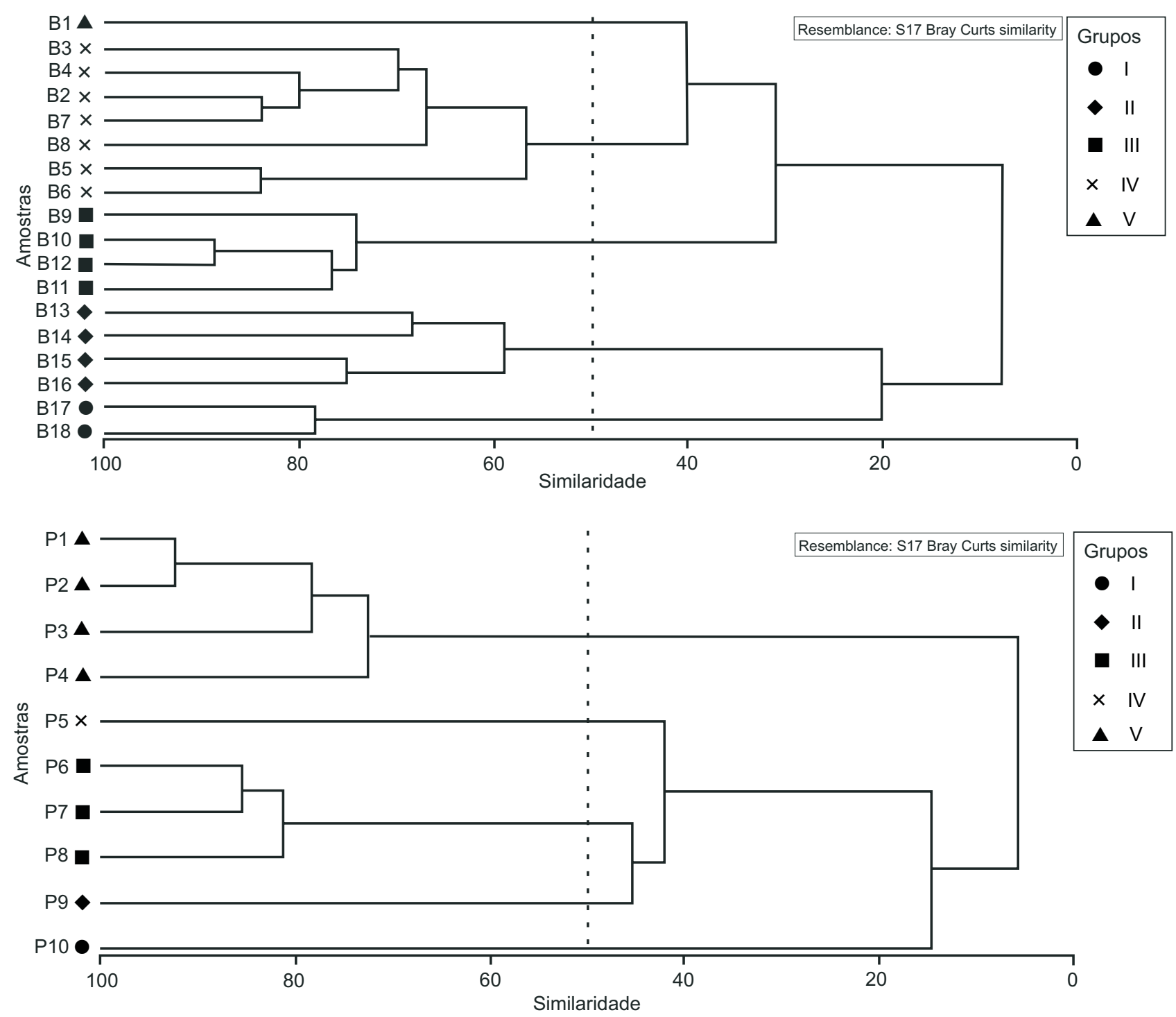

Figura 15. Análise de agrupamento representativa da comunidade bêntica em diferentes alturas (coeficiente de similaridade de BrayCurtis - UPGMA) em ambos os locais estudados (B1 e P1: quadrado inferior nos molhes Barra e Píer = 0,2 a 0,4 m; P10: quadrado superior no Píer = 2,0 a 2,2 m; B18: quadrado superior na Barra: 3,6 a 3,8 m).

spp., Phragmatopoma lapidosa, Centroceras clavulatum e Colisella subrugosa foram os componentes que mais contribuíram. No Grupo V (quadrados inferiores), P. lapidosa contribuiu com a maior similaridade em ambos os locais junto com $P$. perna na Barra e $U$. fasciata no Píer. Vale ressaltar o menor número de espécies que contribuíram para a similaridade dentre os grupos no molhe da Barra.

A análise SIMPER revelou ainda as espécies que mais contribuíram para a distinção entre faixas equivalentes de locais distintos (Tab. V): L. ziczac (58,77\%) e Chthamalus spp. (41,23\%) na faixa superior; Chthamalus spp. (58,00\%), Chaetomorpha sp.
(13,13\%) e L. ziczac $(12,29 \%)$ na faixa intermediária superior; Chthamalus spp. $(45,97 \%)$ e Chaetomorpha sp. $(23,42 \%)$ na faixa intermediária média; $U$. fasciata (26,06\%), Chthamalus spp. $(19,36 \%)$, P. perna $(17,87 \%)$ e C. clavulatum $(13,61 \%)$ na faixa intermediária inferior e $P$. perna $(31,56 \%), U$. fasciata $(31,31 \%)$ e $P$. lapidosa $(23,63 \%)$ na faixa inferior da zona entremarés.

\section{DISCUSSÃO}

A variabilidade espacial em diversas escalas tem sido abordada em muitos estudos promovendo o melhor entendimento da estrutura de comunidades em substratos rochosos no 


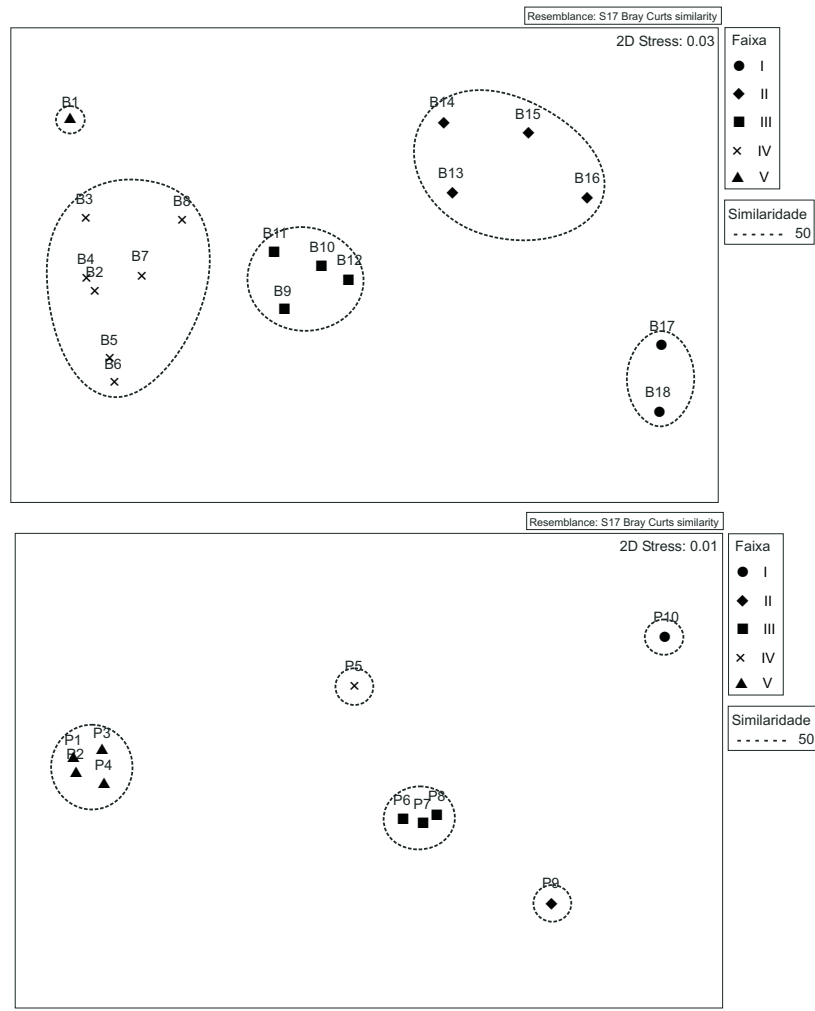

Figura 16. Ordenação MDS da comunidade bêntica em diferentes alturas (coeficiente de similaridade de Bray-Curtis) em ambos os locais estudados. Faixas formadas a partir da análise de agrupamento a um nível de similaridade de 50\% (B1 e P1: quadrado inferior nos molhes Barra e Píer $=0.2$ a 0.4 m; P10: quadrado superior no Píer = 2,0 a 2,2 m; B18: quadrado superior na Barra: 3,6 a 3,8 m)

entremarés (Underwood 1981, Benedetti-Cecchi \& Cinelli 1997, UnderWood \& Chapman 1998a, b, Guichard et al. 2001, Araújo et al. 2005).

No presente estudo foram registradas diferenças com relação à composição de espécies entre a Barra, local mais expos- to, e o Píer, mais protegido. A clorófita Chaetomorpha sp., exclusiva na Barra, é comumente citada na literatura como restrita a locais expostos às ondas (revisado por Oliveira \& Paula 1984, TANAKA et al. 2001, Coutinho 2002). Recifes de areia formados por $P$. lapidosa ocorrem principalmente ao longo da costa tropical nas Américas (FAnTA 1968). Embora tenham sido registrados em ambos os locais de estudo atuando como substrato na porção inferior da zona entremarés, estes ocuparam uma faixa bem mais extensa no Píer. O maior grau de exposição na Barra provavelmente dificulta a expansão dos agregados de arenito que compõe os tubos de P. lapidosa, contudo não exclui a presença da espécie neste ambiente mais batido pelas ondas. Segundo ARAújo et al. (2005), a história de colonização do substrato, a heterogeneidade do ambiente, as interações biológicas e a própria fisiologia dos organismos contribuem conjuntamente para a variabilidade na estrutura da associação encontrada nas diferentes regiões.

Estudos de associações de organismos na zona entremarés focam primariamente na abundância e na distribuição de espécies comuns ou dominantes o que, segundo DAvidson et al. (2004), pode afetar comparações na diversidade. Neste estudo, espécies pouco abundantes como L. ziczac e C. clavulatum contribuíram para as principais diferenças entre os locais. Independente do esquema de zonação adotado é importante que não seja baseado apenas nas espécies dominantes, mas sim no maior número de espécies possível (CoutinHo 1995).

A cobertura de espécies é um bom índice de sua importância (THомаs 1985) e a representação dessa abundância em diagramas, incluindo o nível da maré, auxilia na interpretação do padrão de zonação (СHOI \& KIM 2004). A abundância relativa das espécies variou entre os níveis da zona entremarés, considerando os locais de estudo. Na Barra, todas as espécies ocuparam níveis mais elevados em relação ao Píer, fato explicado pelo batimento mais intenso no primeiro local. Esse efeito, denominado uplift, indica maior grau de exposição às ondas, fazendo com que o borrifo (spray) mantenha a superfície rochosa permanentemente úmida, acima do nível normalmente alcançado pela maré (revisado por BOADEN \& SEED 1985, LiTTLE \& Kitching 1996).

Tabela V. Análise SIMPER com a contribuição das espécies para a dissimilaridade entre os grupos (faixas) equivalentes da Barra (B) e Píer $(P)$, obtidos a partir da análise de agrupamento.

\begin{tabular}{|c|c|c|c|c|c|}
\hline Dissimilaridade entre os grupos (\%) & IB vs IP & IIB vs IIP & IIIB vs IIIP & IVB vs IVP & VB vs VP \\
\hline Chthamalus spp. & 41,23 & 58,00 & 45,97 & 19,36 & \\
\hline Littorina ziczac & 58,77 & 12,29 & & & \\
\hline Chaetomorpha sp. & & 13,13 & 23,42 & & \\
\hline Perna perna & & & 9,28 & 17,87 & 31,26 \\
\hline Ulva fasciata & & & 8,70 & 26,06 & 31,31 \\
\hline Centroceras clavulatum & & & & 13,61 & \\
\hline Phragmatopoma lapidosa & & & & 9,72 & 23,63 \\
\hline Total & 100,00 & 83,51 & 87,37 & 86,61 & 86,50 \\
\hline
\end{tabular}


Assim como a abundância, o número de espécies também variou em relação ao nível da maré e altura do substrato. Diversos autores registraram maior riqueza em níveis inferiores da zona entremarés (Thomas 1985, Boaventura et al. 2002, Good 2004, Araújo et al. 2005). Em ambos os locais em estudo, foram verificadas uma maior riqueza e diversidade de espécies em níveis intermediários, confirmando a Hipótese do Distúrbio Intermediário (ConNell 1978), a qual prevê que a diversidade é maximizada em comunidades sob níveis intermediários de perturbação.

A oscilação periódica da maré favorece a coexistência de organismos em níveis próximos ao intermediário, atingindo o máximo de diversidade. Na porção inferior da zona entremarés, os reduzidos valores de diversidade podem ser atribuídos à elevada turbidez local, dada à proximidade da desembocadura do Rio Paraíba do Sul (63,4 km). Dentre as espécies mais representativas da franja sublitorânea de ambos os locais, destacaram-se as rodófitas. A intensidade luminosa é um fator limitante para a maioria das espécies de macroalgas. Evidências de relações diretas positivas e/ou negativas entre pigmentação, "performance" e distribuição vertical algal são relatadas por KIRK (1994). BoAventura et al. (2002) argumentam claramente que níveis superiores da costa exibem menor diversidade, provavelmente face às condições estressantes nesta altura, o que acarreta em um número bastante reduzido de espécies. Assim como observado por UNDERWOOD (1981) em New South Wales na Austrália, verificou-se uma tendência para o acréscimo da diversidade com o decréscimo da exposição às ondas ao longo da costa, com valores médios superiores no local mais protegido.

Vale ressaltar as expressivas variações de riqueza e diversidade entre as unidades amostrais (perfis) de uma mesma faixa de altura na Barra. Estas variações podem ter ocorrido em função da irregularidade topográfica dos molhes, proporcionando condições ambientais distintas em pequena escala, o que favorece a formação de manchas com diferentes associações de espécies em um mesmo nível. Manchas formando um padrão de distribuição em mosaico também são comumente observadas em Cabo Frio, sudeste do Rio de Janeiro, ocorrendo em escalas que variam desde centímetros a metros (Yoneshigue 1985 apud SAUER-Machado et al. 1992).

A oscilação da maré e o grau exposição às ondas basicamente definem a estrutura da comunidade na zona entremarés (STEPHENSON \& STEPHENSON 1949, LeWIs 1964, UnderWood 1981). A maré proporciona períodos alternados de imersão/emersão, enquanto que o grau de exposição às ondas pode variar de acordo com a altura e o período das ondas tornando a duração e a freqüência de exposição fatores muito importantes. Em locais protegidos, a dependência dos salpicos de água salgada (spray) é um fator relevante para a sobrevivência dos organismos e conseqüentemente para a formação das diferentes faixas. No Píer, ambiente menos exposto, as faixas da zona entremarés mostraram-se mais evidentes do que no sítio Barra, proporcionadas basicamente pela variação da maré.
O padrão de distribuição vertical observado nos dois locais indicou a presença de quatro faixas de organismos na zona entremarés dos respectivos molhes. A orla litorânea foi à única faixa que não mostrou diferenças entre os sítios estudados. GooD (2004) no Caribe, ao comparar essa faixa superior entre locais expostos e protegidos também não registrou diferenças nas associações de organismos, observando que o estresse acentuado se relacionava à exposição extrema ao ar. Na Barra, a orla litorânea mostrou-se três vezes mais ampla (2,6-3,8 m) do que no Píer (1,8-2,2 m). A distribuição dos organismos estava 0,8 m acima do ponto de início no local exposto em relação ao protegido.

Na faixa eulitorânea superior, os organismos precisam suportar grandes períodos de emersão e, portanto, o espaço vazio ainda é muito representativo. No local exposto, a amplitude da faixa $(1,8-2,6 \mathrm{~m})$ mostrou-se um pouco maior que no local protegido (1,2-2,0 m). Na Barra, o bivalve $P$. perna e a clorófita Chaetomorpha sp. apresentaram importante papel na definição dessa zona, reforçando a importância de estudar o maior número de espécies, inclusive aquelas exclusivas como a referida macroalga. Como mencionado, Chaetomorpha é comumente citada como restrita a locais expostos às ondas (revisado por Oliveira \& Paula 1984, Coutinho 2002, Tanaka et al. 2001); ressalta-se que sua morfologia filamentosa proporciona uma menor superfície de contato. A maior abundância relativa do bivalve filtrador $P$. perna no local exposto pode ser resultado de menor predação por Stramonita haemastoma, gastrópode carnívoro bastante comum em fendas no local protegido (observação pesssoal). Segundo Good (2004), o acréscimo da ação das ondas torna a predação progressivamente menos importante que os fatores físicos na organização da comunidade na zona entremarés. O cirripédio Chthamalus spp., comumente encontrado nessa faixa em regiões tropicais e subtropicais em todo o mundo (Coelho et al. 2004), foi o organismo mais importante nesta altura da zona entremarés no Píer.

Na faixa eulitorânea inferior, faixa em que o estresse por dessecação é menos intenso em relação às demais faixas, as associações de organismos foram caracterizadas por espécies comuns aos dois locais, porém com abundância relativa diferenciada. Na Barra, esta faixa ocupou uma grande amplitude da zona entremarés $(0,4-1,8 \mathrm{~m})$, enquanto que no Píer apresentou-se como uma área de transição com apenas $20 \mathrm{~cm}$ de amplitude (1,0-1,2 m). Comparando-se os dois locais, diversas espécies foram responsáveis pela diferença dessa faixa como Chthamalus spp., C. clavulatum e P. lapidosa (Píer) e P. perna e $U$. fasciata (Barra).

Na franja sublitorânea o período de imersão é mais prolongado e geralmente esta faixa é ocupada em sua maior parte por macroalgas (sensu Lewis 1964). As macroalgas foram mais abundantes no local protegido, enquanto no exposto os molhes apresentavam-se recobertos pelos recifes arenosos de $P$. lapidosa. SAuer-Machado et al. (1992) citam este poliqueta em costão rochoso moderadamente protegido em Búzios, sudeste do Rio de Janeiro. 
Nesta faixa também se observou diferença entre os locais com relação à abundância de $P$. perna, predominante no de maior batimento, e $U$. fasciata e $H$. musciformis, predominatemente no local protegido. Ao longo da costa portuguesa, os mexilhões também ocorrem na porção inferior do médiolitoral (= região eulitorânea) de costões mais expostos (BoAventura et al. 2002).

As diferenças encontradas nos padrões de zonação na zona entremarés nas superfícies dos molhes estudados são atribuídas principalmente a diferença no grau de exposição às ondas em que estão submetidos, como a altura diferenciada ocupada pelas espécies representativas bem como a extensão de suas respectivas faixas. O efeito uplift relacionado à ampliação da zona entre marés foi identificado no local mais exposto, em que a orla litorânea apresentou-se três vezes mais extensa. As associações de espécies nas demais faixas apresentaram-se diferenciadas tanto na composição de espécies como em suas respectivas abundâncias relativas. O presente estudo complementa a base de dados sobre a comunidade bêntica da zona entremarés em substratos consolidados na costa norte do estado do Rio de Janeiro.

\section{AGRADECIMENTOS}

Aos pesquisadores Renato Crespo Pereira (Departamento de Biologia Marinha, UFF) e Ronaldo Novelli (Laboratório de Ciências Ambientais, UENF) pela identificação das algas e moluscos, respectivamente. Á Fundação de Amparo à Pesquisa do Estado do Rio de Janeiro pelo auxílio à pesquisa (Processo E26-171.313/04).

\section{LITERATURA CITADA}

Araújo R.; I. Bárbara; I. Sousa-Pinto \& V. Quintino. 2005. Spatial variability of intertidal rocky shore assemblages in the northwest coast of Portugal. Estuarine, Coastal and Shelf Science 64: 658-670.

Benedetti-Cecchi, L. 2000. Predicting direct and indirect effects during succession in a midlittoral rocky shore assemblage. Ecology Monograph 70: 45-72.

Benedetti-Cecchi, L. \& F. Cinelli. 1997. Spatial distribution of algae and invertebrates in the rocky intertidal zone of the Strait of Magellan: are patterns general? Polar Biology 18: 337-343.

BOADEN, P.J.S. \& R. SEED 1985. An introduction to coastal ecology. Glasgow, Blacckie and Son, 218p.

Boaventura, D.; P. Ré; L.C. Fonseca \& S.J. Hawkins. 2002. Intertidal Rocky Shore Communities of the Continental Portuguese Coast: Analysis of Distribution Patterns. Marine Ecology 23 (1): 69-90.

Bulleri, F. \& M.G. Chapman. 2004. Intertidal assemblages on artificial and natural habitats in marinas on the north-west coast of Italy. Marine Biology 145: 381-391.

CHor T.S. \& K.Y. KIM. 2004. Spatial pattern of intertidal macro- algal assemblages associated with tidal levels. Hydrobiologia 512: 49-56.

Clarke K.R. \& R.M. Warwick. 2001. Change in marine communities: an approach to statistical analysis and interpretation. Plymouth, PRIMER-E, UK, $2^{\text {nd }}$ ed., 172p.

Collho, P.A.; D. De O. Tenório; M.M. Ramosporto \& L.S. Rosa. 2004. A fauna bêntica do Estado de Pernambuco. p. 477527. In: E. EskinaZi-LeçA; S. Neumann-Leitão \& M. F. Costa (Eds). Oceanografia: um cenário tropical. Recife, Edições Bagaço, 761p.

Connel J.H. 1978. Diversity in tropical rain forest and coral reefs. Science 199: 1302-1310.

Coutinho, R. 1995. Avaliação crítica das causas da zonação dos organismos bentônicos em costões rochosos. Oecologia Brasiliensis 1: 259-271.

Coutinho, R. 2002. Bentos de Costões Rochosos, p.147-157. In: R.C. Pereira \& A. Soares-Gomes (Eds). Biologia Marinha. Rio de Janeiro, Interciência Press, 382p.

Davidson I.C.; A.C. Crook \& D.K.A. Barnes. 2004. Quantifying spatial patterns of intertidal biodiversity: is movement important? Marine Ecology 25 (1): 15-34.

FANTA, E.S. 1968. Sobre a biologia e ecologia de Phragmatopoma lapidosa (Sabellariidae, Polychaeta). Ciência e Cultura 20: 459-460.

Gevertz, R. 1995. Em busca do conhecimento ecológico: uma introdução à metodologia. São Paulo, Edgard Blucher, $128 \mathrm{p}$.

Good, T.P. 2004. Distribution and abundance patterns in Caribbean rocky intertidal zones. Bulletin of Marine Science 74 (2): 459-468.

Guichard, F.; E. Bourget \& J.L. Robert. 2001. Scaling the influence of topographic heterogeneity on a intertidal benthic communities: alternate trajectories mediated by hydrodynamics and shading. Marine Ecology Progress Series 217: 27-41.

HarPer, J.R.; D.E. Howes \& P.D. Reimer. 1991. Shore-zone mapping system for use in sensitity mapping and shore countermeasures. Proceedings of the $14^{\text {th }}$ Artic and Marine Oilspill Program (AMOP), Environment Canada 1: 509-23.

JACOBI, C.M. \& R. LANGevin. 1996. Habitat geometry of benthic substrata: effects on arrival and settlement of mobile epifauna. Journal of Experimental Marine Biology and Ecology 206: 39-54.

KIRK, J.T.O. 1994. Light and Photosynthesis in Aquatic Ecosystems. Cambridge, Cambridge University Press, 509p.

Kohler, K.E. \& S.M. Gill. 2006. Coral Point Count with Excel extensions (CPCe): A Visual Basic program for the determination of coral and substrate coverage using random point count methodology. Computers and Geosciences 32 (9): 1259-1269.

LEWIS, J.R. 1964. The ecology of rocky shore. London, English University Press, 300p.

Little, C. \& J.A. Kiching. 1996. The biology of rocky shores. 
Oxford, Oxford University Press, 240p.

Macedo I.M.; B.P. Masi \& I.R. Zalmon. 2006. Comparision of rocky intertidal community sampling methods at the northern coast of Rio de Janeiro state, Brasil. Brazilian Journal of Oceanograph 54 (2/3): 147-154.

Menge B.A.; G.W. Allison; C.A. Blanchette; T.M. Farrell; A.M. Olson; T.A. Turner \& P.V. Tamalen. 2005. Stasis or kinesis? Hidden dynamics of a rocky intertidal macrophyte mosaic reveled by a spatially explicit approach. Journal of Experimental Marine Biology and Ecology 314: 3-39.

Moore, P.G. \& R. SEED. 1985. The ecology of rocky coasts. London, Hodder and Stoughton, 467p.

Oliveira, E.C. \& E.J. Paula. 1984. Aspectos da distribuição vertical e variação sazonal de comunidades da zona das marés em costões rochosos do litoral norte de São Paulo. Revista Brasileira de Biologia 147: 44-71.

PAUlA, E.J. 1987. Zonação nos costões rochosos: região entremarés. Simpósio sobre ecossistemas da costa sul e sudeste brasileira: síntese dos conhecimentos. Cananéia 1: 266-288.

Preskitt, L.B.; P.S. Vroom \& C.M. Smith. 2004. A Rapid Ecological Assessment (REA) Quantitative Survey Method for Benthic Algae Using Photoquadrats with Scuba. Pacific Science 58 (2): 201-209.

Sauer-Machado, K.R.S.; A.R.O. Chapman \& R. Coutinho. 1992. Patch structure in a tropical rocky shore community in Brazil: A mosaic of sucessional states? Ophelia 35 (3): 187-195.

Schoch, G.C. \& M.N. Dethier 1997. Analysis of shoreline classification and bio-physical data for Carr inlet. Olympia,
Report for Washington State Department of Natural Resources, Aquatic Resources Division, 198p.

Stephenson, T.A. \& A. Stephenson. 1949. The Universal feature of zonation between tide-marks on rocky coasts. Journal of Ecology 37: 289-305.

Tanaka, M.O.; T.E.M. Duque-Estrada \& C.A. Magalhães. 2001. Dynamics of the Acmaeid limpet Collisella subrugosa and vertical distribution of size and abundance along a wave exposure gradient. Journal of Molluscan Studies 68: 5564.

Tномаs, M.L.H. 1985. Littoral community and zonation on the rocky shores of Bermuda. Bulletin of Marine Science 37: 857-870.

UNDERWOOD, A.J. 1981. Structure of a rocky intertidal community in New South Wales: Patterns of vertical distribution and seasonal changes. Journal of Experimental Marine Biology and Ecology 51 (7): 57-85.

Underwood, A.J. \& M.G. Chapman. 1998a. Variation in algal assemblages on wave-exposed rocky shores in New South Wales. Marine and Freshwater Research 49: 241-254.

Underwood, A.J. \& M.G. Chapman. 1998b. Spatial analyses of intertidal assemblages on sheltered rocky shores. Austral Ecology 23: 138-157.

VALENTIN, J.L. 2000. Ecologia Numérica - uma introdução à análise multivariada de dados ecológicos. Rio de Janeiro, Ed. Interciência, $117 \mathrm{p}$.

ZAR, J.H. 1984. Biostatistical analysis. New Jersey, Prentice Hall, $718 \mathrm{p}$.

Submitted: 19.IX.2007; Accepted: 28.XI.2008.

Editorial responsibility: Paulo da Cunha Lana 